\title{
Estilo de vida, prática de exercício físico e dores musculoesqueléticas em idosas fisicamente ativas
}

\author{
Érico Felden Pereira*, Clarissa Stefani Teixeira*", Luciane Sanchotene Etchepare Daronco"**, \\ Marco Aurélio Acosta***
}

\section{Resumo}

Este estudo buscou investigar as características de estilo de vida, práticas de atividades físicas e dores musculoesqueléticas de idosas ativas. Foram avaliadas 71 idosas por meio de um questionário com perguntas fechadas e semiabertas e questionário de Corlett e Bishop (1976) para identificação das queixas musculoesqueléticas. Os resultados indicam que, além da atividade de ginástica, $71,3 \%$ das idosas realizam hidroginástica; $88,89 \%$ dos idosos procuraram os exercícios físicos pelo fato de melhorar a qualidade de vida. A frequência semanal de realização das atividades é de uma vez na semana para 67,1\% das idosas e duas vezes na semana para $32,9 \% ; 68,2 \%$ das idosas passam a maior parte do tempo caminhando. As queixas foram identificadas principalmente na região cervical $(64,7 \%)$, seguida dos braços $(58,8 \%)$ e da região lombar $(47,1 \%)$. Um fato a destacar é o uso de medicamentos por $50 \%$ das idosas investigadas para o combate dos sintomas de dor e desconforto musculoesquelético. São necessárias intervenções com programas de educação em saúde e de exercícios físicos voltados à melhora das queixas apresentadas pelas idosas, agindo como atividade preventiva e melhorando a qualidade de vida.

Palavras-chave: Idoso. Saúde do idoso. Dor.

\section{Introdução}

A totalidade dos fatores envolvidos no processo de envelhecimento não é bem conhecida. Sabe-se que o envelhecimento é biologicamente programado e marcado por condições genéticas limitadoras na replicação de genes no interior das células. (BEE, 1997). O processo de

* Professor de educação física. Doutorando em Educação Física na Universidade Federal do Paraná. Bolsista Capes. Endereço para correspondência: Otacílio Chaves, 253. CEP: 97045-360. Santa Maria - RS. E-mail: ericofelden@gmail.com.

** Professora de educação física. Doutoranda em Engenharia de Produção na Universidade Federal de Santa Catarina. Bolsista CNPq.

*** Professora de educação física. Doutora em Ciência do Movimento Humano pela Universidade Federal de Santa Maria. Professora da Universidade Federal de Santa Maria.

***** Professor de educação física. Doutor em Ciência do Movimento Humano pela Universidade Federal de Santa Maria. Professor da Universidade Federal de Santa.

$\hookrightarrow$ Recebido em dezembro de 2008 - Avaliado em setembro de 2009.

$\rightarrow$ doi:10.5335/rbceh.2009.033 
envelhecimento é influenciado de forma importante pelos comportamentos em relação a aspectos como tabagismo, etilismo e alimentação (HAYFLICK, 1997) e relacionamentos interpessoais e apoio familiar. (RAMOS, 2002). Além desses fatores, a atividade física tem sido apontada como fator de proteção para problemas mentais nos idosos. (BENEDETTI et al., 2008).

Com o aumento da longevidade, maiores prevalências de doenças crônicodegenerativas vêm sendo verificadas, trazendo consequências econômicas e sociais. Nesse contexto, observa-se um aumento crescente na implantação de programas de atividades físicas em todo o mundo como meio de promoção de hábitos saudáveis. (BENEDETTI; GONÇALVES; MOTA, 2007). Até 2025, possivelmente, o país terá a sexta maior população de idosos do mundo, ou seja, mais de 32 milhões de pessoas com sessenta anos de idade ou mais. Assim, faz-se necessária a implantação de programas voltados à qualidade de vida dos idosos, nos quais profissionais das mais diversas áreas possam atuar com o intuito de alcançar metas para a melhoria não só da saúde, mas também da qualidade de vida e longevidade.

Nesse contexto, análises acerca das diferentes características dos idosos inseridos em grupos de atividades físicas, com levantamento de prevalências e comportamentos, podem fomentar discussões para planejamentos adequados de atividades, bem como de políticas públicas para essa população. Dessa forma, este estudo objetivou investigar algumas características de idosos fisi- camente ativos, como o estilo de vida, as práticas de exercícios e as queixas musculoesqueléticas.

\section{Procedimentos metodológicos}

Fizeram parte do estudo idosos praticantes da modalidade de ginástica ofertada por uma instituição federal de ensino superior, do gênero feminino, com idades a partir dos sessenta anos. Formaram o grupo de investigação 71 idosas com idade de 69,7 e desvio-padrão de 5,8 anos, massa corporal de 64,7 e desvio-padrão de $11,7 \mathrm{~kg}$ e estatura de 1,6 e desvio-padrão de $0,1 \mathrm{~m}$.

Todas as idosas responderam a um questionário com perguntas fechadas e semiabertas relacionadas aos hábitos diários, prática e frequência semanal de exercícios físicos, aos motivos que as levam a essas práticas, utilização de medicamento, queixas musculoesqueléticas e tratamentos realizados para essas. O estudo foi aprovado pelo Comitê de Ética da instituição de origem, sob protocolo $\mathrm{n}^{\circ}$ 0044.0243.000-07, considerando os princípios éticos para a realização de estudos com seres humanos da Resolução 196/96 do Conselho Nacional da Saúde.

Para a verificação dos sintomas musculoesqueléticos foi aplicado o questionário para identificação da dor nas partes do corpo adaptado do instrumento desenvolvido por Corlett e Bishop (1976), que consiste numa folha com um mapa corporal dividido em 28 partes. As queixas musculoesqueléticas foram classificadas como sintomas de dor, desconforto, formigamento ou dormência nas regiões corporais. (BARROS; ALEXANDRE, 
2003). Os dados foram analisados por meio do percentual das respostas.

\section{Resultados e discussões}

Kuwano e Silveira (2002) evidenciaram o aumento da demanda de idosos que procuram a atividade física e citam as preocupações relacionadas ao monitoramento e pesquisas com a população da terceira idade a fim de obter maiores informações e subsídios para a proposição de atividades adequadas, seguras e eficientes para o idoso.

De forma geral, o exercício físico é visto pelos profissionais da área da saúde como fator preventivo e, até mesmo, de reabilitação. A conscientização quanto aos benefícios que a prática regular e bem orientada pode trazer é vista atualmente no Brasil, uma vez que os dados científicos ilustrados nas pesquisas apontam para a prescrição médica como um dos fatores para se procurar algum tipo de exercício. (ANDRADE et al., 2000). No presente estudo, $88,9 \%$ dos idosos procuraram os exercícios físicos para melhorar sua qualidade de vida; $64,7 \%$, simplesmente pelo fato de gostar; $35,3 \%$, por orientação médica e $11,1 \%$, para sociabilização.

Segundo Soares (2002), mesmo que existam alterações da vida no processo do envelhecimento, o conceito de que a velhice é incapaz e decadente não é justificado nem pode ser afirmado. Com o passar do tempo, a iniciativa, o entusiasmo e a motivação tendem a diminuir, sendo necessários ao idoso estímulos, para não se isolar socialmente. As alterações que ocorrem com o processo de envelhecimento podem ser amenizadas com uma adequada preparação para que a velhice seja uma fase agradável e o processo de envelhecimento se torne algo natural, sem grandes conflitos, aumentado, assim, a vontade de viver, o que salienta a necessidade da busca pela qualidade de vida dos idosos.

Para Silva et al. (2007), o sedentarismo, aliado à idade cronológica, induz a efeitos negativos sobre a capacidade funcional de mulheres acima de 51 anos. Portanto, a melhor alternativa para se terem condições de boa saúde e qualidade de vida é a prática de exercícios físicos.

Pinho et al. (2006) encontraram num grupo de idosas do gênero feminino $74 \%$ delas praticando exercícios físicos (hidroginástica) com o objetivo de melhorias relacionadas à saúde; $10 \%$, por socialização (convívio com outras pessoas); $6,7 \%$, para preencher o tempo disponível; 3,3\%, por lazer/gostar; $1,7 \%$, para diminuir a depressão; $1,7 \%$, para ser ágil nas tarefas cotidianas e $1,7 \%$, por problemas emocionais.

Segundo Mazo (2003), o público da terceira idade está cada vez mais ativo, participando de diversas atividades físicas e obtendo, assim, vários benefícios físicos e psíquicos. Entre essas práticas é possível citar a caminhada, a hidroginástica e os exercícios resistidos como os mais praticados pelos idosos. (CRUZ et al., 2006). Essas indicações são confirmadas pelo presente estudo, uma vez que todos (100\%) os idosos avaliados realizam atividades de ginástica. Desses, além desta atividade, $71,3 \%$ praticam hidroginástica e $67,1 \%$ caminhada e/ou 
hidroginástica. Apenas $28,8 \%$ dos idosos pesquisados não praticam outra modalidade além da de ginástica.

Teixeira et al. (2008) relataram que práticas de atividades físicas distintas na terceira idade, como hidroginástica e ginástica, atuam de forma diferenciada na manutenção do equilíbrio corporal quando a direção médio-lateral foi avaliada. Os autores constataram menores instabilidades nas mulheres idosas praticantes de ginástica, o que demonstra um dos benefícios de se manter fisicamente ativo, já que os problemas de desequilíbrio nos idosos são comuns.

Um estilo de vida sedentário leva a um declínio adicional na capacidade e eficiência dos sistemas e aparelhos do corpo. Isso intensifica o processo de envelhecimento e, em consequência, produz uma aparência mais debilitada. Rosa et al. (2008) observaram que um período de 12 semanas de destreino em atividades de hidroginástica e natação acarreta perdas no índice de aptidão funcional geral e na coordenação de idosas. Esses resultados, segundo os autores, levaram a que um maior número de idosas passasse a ter aptidão considerada fraca. Teixeira (2008) também relatou pioras em relação ao equilíbrio corporal e uma tendência de reincidência nas queixas de tontura e piora da qualidade de vida de idosos após período de dois meses sem prática de hidroginástica.

Um estilo de vida ativo melhora a capacidade e a eficiência desses sistemas, podendo retardar ou até reverter alguns dos efeitos do envelhecimento. (WAGORN; THÉBERGE; ORBAN, 1993). Para Pinho et al. (2006), pessoas que praticam algum tipo de exercício tendem a ser menos deprimidas do que as que não os praticam, o que, mais uma vez, aponta a importância de se manter fisicamente ativo.

Idosos praticantes de exercícios físicos conseguem melhoras na saúde, qualidade de vida e bem-estar geral. Teixeira (2008), avaliando um grupo de idosos com e sem queixas de tontura, observou melhoras com 12 sessões de hidroginástica nos aspectos físicos, emocionais e funcionais da qualidade de vida, além da redução das queixas de tontura. Neste estudo também foi observada melhora no equilíbrio corporal e, consequentemente, nos sistemas visual, proprioceptivo e vestibular. Melhoras na estabilidade postural também foram relatadas com a prática de atividade proposta, indicando a necessidade de um estilo de vida ativo. No estudo de Mann et al. (2008) a hidroginástica foi o exercício recomendado para os idosos, uma vez que esses não se mostraram diferentes aos indivíduos mais novos em relação ao equilíbrio corporal.

A prática de exercícios físicos foi considerada como benéfica por Pacheco et al. (2005). Os autores relataram melhoras relacionadas à qualidade de vida e à performance motora de indivíduos idosos. Para Kuwano e Silveira (2002) idosos praticantes há mais de um ano melhoraram em relação aos aspectos físico, psíquico e social, recebendo o conceito de "muito bom", ou seja, classificação máxima de acordo com a escala proposta pelo estudo.

Com a prática de exercícios físicos em grupos de convivência, foram 
observadas por Bulsing et al. (2007) ressocialização, aumentando o grupo de amizades, e melhora da autoestima, dando um novo sentido para a vida. Areosa e Ohlweiler (2000) afirmam que a inserção nos grupos de terceira idade, além de redimensionar a identidade, traz satisfação pessoal, aumento dos relacionamentos e reconhecimento do outro perante o grupo.

Além dos benefícios psicossociais, a prática de exercícios físicos é relacionada a melhoras nas condições de saúde dos idosos. (GIATTI; BARRETO, 2003). Para Rasia et al. (2007), a prática de exercícios físicos é moderadora do peso corporal. Logo, manter-se fisicamente ativo pode evitar algumas das consequências da obesidade para a saúde. Cabrera e Jacob Filho (2001) identificaram correlações entre os indicadores antropométricos e as alterações metabólicas associadas à obesidade. Os resultados revelam diferenças em relação ao sexo, com os homens apresentando menor prevalência de obesidade e maior associação entre índice de massa corporal (IMC), ou relação cintura quadril (RCQ), e os fatores de riscos relacionados à gordura corpórea. Para Silva et al. (2007), o valor do IMC em idosos é um dos fatores predisponentes à incapacidade funcional.

Outra questão importante relacionada à manutenção de um estilo de vida fisicamente ativo é o tempo de realização dessas práticas. Nesse sentido, houve atividade há mais de quatro anos para $64,5 \%$ das idosas avaliadas e de seis meses para $33,5 \%$ das idosas. Pode-se notar aqui que a maioria das avaliadas já pratica exercícios há bastante tempo, conseguindo, assim, manter-se na atividade, o que é um fator que contribui para a saúde. Teixeira, Pereira e Acosta (2003), investigando os participantes de um evento desenvolvido para indivíduos da terceira idade, inferiram as relações entre prática de exercícios e saúde, uma vez que mais da metade dos 1.066 idosos investigados afirmou não possuir nenhum tipo de patologia.

Nahas (1997) e Pieron (2004) comentam que estudos têm cada vez mais comprovado a importância de a pessoa se manter em atividades que resultem em um gasto calórico ao longo do dia, ao invés de uma única atividade intensa e concentrada. Segundo Vargas e Vargas Neto (2000), ao chegar à terceira idade, os hábitos próprios têm influenciado na expectativa de vida. Assim, quanto mais os idosos seguirem um estilo de vida saudável, mais aumentará a longevidade, permitindo-lhes a continuidade e autonomia em suas habilidades físicas e mentais, o que contribuirá para uma vida mais longa e feliz. Estilo de vida mais saudável pode permitir que um maior número de jovens e adultos de meia idade da atualidade mantenha um nível elevado de funcionamento físico em boa parte da terceira idade.

Com relação à frequência semanal, a maioria dos idosos realiza atividades regulares, pelo menos, uma vez na semana $(67,1 \%)$ e $32,9 \%$, duas vezes; $68,2 \%$ dos idosos passam a maior parte do tempo caminhando e $31,8 \%$ passam a maior parte do tempo sentados; $100 \%$ dos idosos realizam serviços leves e $69,4 \%$ afirmam que realizam em seu dia a dia serviços pesados. Assim, percebe-se que 
a maior parte dos idosos, além das atividades desenvolvidas pelo grupo, procura manter uma vida ativa praticando atividades físicas durante o dia.

Quanto à atividade física, Pascoal, Santos e Van den Broek (2006) avaliaram idosos de duas regiões, verificando que $94 \%$ dos idosos de um dos grupos foram considerados ativos, visto que $88,89 \%$ praticavam exercícios com uma frequência semanal de duas vezes por semana ou mais. No outro grupo avaliado, 70,6\% faziam regularmente algum tipo de exercício físico, dos quais $67,6 \%$ praticavam-nos duas vezes por semana ou mais. Neste estudo o tempo médio de duração da prática para os dois grupos foi de uma hora.

Uma das questões que atuam contra os benefícios dos exercícios físicos quando iniciados pelo idoso é a dor musculoesquelética. Porém, nem sempre a dor pode ser atribuída aos exercícios físicos, visto que muitas vezes está intimamente relacionada aos hábitos do dia a dia. No presente estudo, todos os idosos afirmam sentir dor em alguma parte do corpo, como dores na região cervical $(64,7 \%)$, região lombar $(47,1 \%)$, região dorsal do tórax $(23,5 \%)$, nos braços $(58,82 \%)$, nas pernas $(41,2 \%)$ e nos pés $(11,8 \%) ; 85,7 \%$ dos idosos afirmam sentir mais dores no período da noite e, apra $14,3 \%$, durante o dia a dor se apresenta mais forte. Nenhum dos idosos avaliados relacionou a prática do exercício físico com a presença da dor.

Rasia et al. (2007) também identificaram presença de dor e desconforto musculoesquelético em alguma parte do corpo de mulheres pós-menopausa com idades de 58,4 e desvio-padrão de 3,6 anos. A dor foi referida por $67 \%$ das participantes do estudo, ao passo que $33 \%$ delas não a apresentaram. Ao identificar os locais de maiores queixas, a coluna lombar mostrou-se uma das principais regiões com problemas, com $43,7 \%$, seguida de queixas localizadas no joelho, com 40,6\%; no tornozelo/pé, com $28,1 \%$; no ombro, com $28,1 \%$, e no quadril, com $21,8 \%$ das respostas.

No combate da dor, $50 \%$ dos idosos que compõem este estudo fazem uso de algum tipo de medicamento. Porém, mesmo que o uso de medicamentos na terceira idade seja comum, sua utilização é um dos fatores de riscos relacionados às quedas que provocam fraturas. (HANRA; RIBEIRO; MIGUEL, 2007). De forma geral, a prática de atividade física regular é uma forma de prevenir quedas em pessoas idosas. Idosos sedentários têm menor mobilidade e maior propensão a quedas em comparação a idosos que praticam atividade física regularmente. (GUIMARÃES et al., 2004).

Ao analisar a interferência da dor nas atividades diárias (domésticas, ocupacionais e lazer), verificou-se que a dor e desconforto musculoesquelético interferem acentuadamente nas atividades domésticas, fato relatado por $53,1 \%$ das mulheres participantes do estudo, seguida pela interferência nas atividades de lazer, com 25\%. (RASIA et al., 2007).

Conte e Lopes (2005) encontraram a dor e o desconforto como impedimentos para a realização das tarefas diárias em $40 \%$ das idosas estudadas. De maneira geral, o estudo mostrou associação entre o nível de atividade física e a qualidade 
de vida. As idosas ativas se mostraram com menos dor e com mais energia para o dia a dia, estando mais satisfeitas com a capacidade de locomoção, com a capacidade de trabalho e pela desenvolver atividades diárias, sem necessidade de tratamento médico.

No presente estudo, $81,3 \%$ dos idosos já realizaram tratamento para distúrbios musculoesqueléticos. Segundo Lorda e Sanchez (1995), o envelhecimento traz dois tipos de transtornos: diminuição do tônus e redução dos níveis de força. Observa-se uma redução na massa muscular em 10-20\% por volta dos 65 anos, tanto no tamanho da fibra como em número. (MATSUDO, 1991). Para Soares (2002), as articulações também sofrem grandes mudanças, pois perdem a mobilidade e a elasticidade. Dessa forma, podem levar a lesões degenerativas do aparelho capsular, conhecido como artrose, o que pode afetar um número alto de articulações, com maior frequência nos joelhos, quadris, dedos, ombros, coluna lombar e cervical.

Porém, há evidências na literatura sobre a melhora da força muscular com a prática de exercícios físicos. Brown et al. (2000) aplicaram um protocolo de exercícios que continha treinos de transferência e equilíbrio, alongamentos e fortalecimento muscular de membros inferiores e superiores em 48 idosos (homens e mulheres), com média de idade de 83,0 e desvio-padrão de 4,0 anos, durante três meses, com frequência de três vezes por semana. $\mathrm{O}$ grupo de controle foi composto por 39 idosos com a mesma média de idade, os quais realizaram voluntariamente apenas exercícios de alongamento. Dentre os resultados obtidos, verificou-se que, concomitantemente com o aumento de $9 \%$ da força muscular, ocorreu melhora na capacidade física dos indivíduos do grupo experimental, inclusive no equilíbrio dinâmico e estático, o que sugere que o programa de exercícios proposto foi efetivo para aumentar o desempenho funcional dos idosos, uma vez que o grupo de controle não apresentou os mesmos resultados. Cao et al. (2007) também encontraram efeitos positivos nas forças dos membros inferiores de 20 idosos com idades entre 65 a 79 anos realizados duas vezes por semana. Hess e Woollacott (2005), igualmente, indicaram que exercícios intensos de treinamento de força podem, efetivamente, reforçar os músculos dos membros inferiores de idosos com défice de equilíbrio, resultando em significativo aumento na capacidade funcional do equilíbrio e na diminuição do risco de quedas.

\section{Considerações finais}

Este estudo buscou investigar algumas características de estilo de vida e de práticas de exercícios físicos de mulheres idosas. Os resultados indicam que as idosas procuram se manter ativas realizando uma ou mais modalidades, sendo a ginástica e a hidroginástica as principais. A busca da qualidade de vida foi a que mais se destacou como o motivo que leva as idosas a prática de exercícios físicos regulares.

Mesmo que as idosas possuem um estilo de vida fisicamente ativo, podemse relacionar as queixas musculoesqueléticas como um dos fatores preocupantes 
nesta população, assim como o uso de medicamentos para a atenuação desses sintomas.

Lifestyle, practice physical exercise and musculoskeletal pain in elderly physically active

\section{Abstract}

This study aimed to investigate the characteristics of lifestyle, physical activity and musculoskeletal pain in older active; 71 elderly was evaluated through a questionnaire with closed and semi-open questions and the questionnaire of Corlett and Bishop (1976) for identification of musculoskeletal complaints. The results indicate that besides of gymnastics activity, $71,3 \%$ of the elderly realized hidrogymnastic. $88.89 \%$ of the elderly looked for physical exercise to improve the quality of life. The weekly frequency of activities realization is once a week for $67,1 \%$ of the elderly and twice in the week to $32,9 \% ; 68,2 \%$ of the elderly spend most of their time walking. The complaints have been identified mainly in the neck $(64,7 \%)$, followed by the arms $(58,8 \%)$ and lumbar region $(47,1 \%)$. A fact the highlight is the use of medicines by $50 \%$ of elderly investigated to combat the symptoms of pain and discomfort musculoskeletal. Its necessary interventions with programs of health education and physical exercises aimed at improvement of the complaints showed by the elderly, acting as a preventive activity and improving the quality of life.

Key words: Aged. Health of the elderly. Pain.

\section{Referências}

ANDRADE, E. L. et al. Barries and motivational factors for physical activity adherence in elderly people in developing country. Medicine and Science in Sports and Exercise, v. 33, supl, 7, p. 141, 2000.

AREOSA, S. V. C.; OHLWEILER, Z. N. C. O idoso e os grupos de convivência no município de Santa Cruz do Sul: dados de pesquisa. Revista Redes, Santa Cruz do Sul, v. 5, n. 1, p. 179-187, jan./abr. 2000.

BARROS, E. N. C.; ALEXANDRE, N. M. C. Cross-cultural adaptation of the Nordic musculoskeletal questionnaire. International Nursing Review, v. 50, n. 2, p. 101-108, 2003.

BEE, H. O ciclo vital. Porto Alegre: Artes Médicas, 1997.

BENEDETTI, T. R. B. et al. Atividade física e estado de saúde mental de idosos. Revista de Saúde Pública, Rio de Janeiro, v. 42, n. 2, p. 302-307, 2008.

BENEDETTI, T. R. B.; GONCALVES, L. H. T. MOTA, J. A. P. S. Uma proposta de política pública de atividade física para idosos. Texto e Contexto em Enfermagem, Florianópolis, v. 16, n. 3, p. 387-398, 2007.

BROWN, M. et al. Low-intensity exercise as a modifier of physical frailty in older adults. Archives of Physical Medicine and Rehabilitation, v. 81, n. 7, p. 960-965, 2000.

BULSING, F. L. et al. A influência dos grupos de convivência sobre a auto-estima das mulheres idosas do município de Santa Cruz do Sul - RS. Revista Brasileira de Ciência do Envelhecimento Humano, Passo Fundo, v. 4, n. 1, p. 11-17, jan./jun. 2007.

CABRERA, M. A. S.; JACOB FILHO, W. Obesidade em idosos: prevalência, distribuição e associação com hábitos e co-morbidades. Arquivos Brasileiros de Endocrinologia \& Metabologia, São Paulo, v. 45, n. 5, p. 494-501, 2001. 
CAO, Z. B. et al. The effect of a 12 -week combined exercise intervention program on physical performance and gait kinematics in community-dwelling elderly women. J. Physiol. Anthropol., v. 26, n. 3, p. 325-332, 2007.

CONTE, E. M. T.; LOPES, A. S. Qualidade de vida e atividade física em mulheres idosas. Revista Brasileira de Ciências do Envelhecimento Humano, Passo Fundo, v. 2, n. 1, p. 61-75, jan./jun. 2005.

CORLETT, E. N.; BISHOP, R. P. A technique for assessing postural discomfort. Ergonomics, v. 19, n. 2, p. 175-182, 1976.

CRUZ, P. G. et al. Relação entre qualidade de vida e exercício físico na terceira idade. 2006. Disponível em: <www.wgate.com.br/ conteudo/medicinaesaude/fisioterapia/variedades/rel_exerc_terceira_idade_heloisa. htm>. Acesso em: 3 ago. 2007.

GIATTI, L.; BARRETO, S. M. Saúde, trabalho e envelhecimento no Brasil. Cadernos de Saúde Pública, Rio de Janeiro, v. 19, n. 3, p. 759-771, 2003.

GUIMARÃES, L. H. C. T. et al. Comparação da propensão de quedas entre idosos que praticam atividade física e idosos sedentários. Neurociência, São Paulo, v. 12, n. 2, p. 68-72, 2004.

HAMRA, A.; RIBEIRO, M. B.; MIGUEL, O. F. Correlação entre fratura por queda em idosos e uso prévio de medicamentos. Acta Ortopética Brasileira, São Paulo, v. 15, n. 3, p. 43-145, 2007.

HAYFLIK, L. Como e por que envelhecemos. Rio de Janeiro: Campus, 1997.

HESS, J. A.; WOOLLACOTT, M. Effect of high-intensity strength-training on functional measures of balance ability in balanceimpaired older adults. Journal of Manipulative and Physiological Therapeutics, v. 28, n. 8, p. 582-590, 2005.

KUWANO, V. G.; SILVEIRA. A. M. A influência da atividade física sistematizada na autopercepção do idoso em relação às atividades da vida diária. Revista da Educação Física / UEM, Maringá, v. 13, n. 2, p. 35-39, 2002.
LORDA, C. R.; SANCHEZ, C. D. Recreação na terceira idade. Rio de Janeiro: Sprint, 1995.

MANN, L. et al. Investigação do equilíbrio corporal em idosos. Revista Brasileira de Geriatria e Gerontologia, Rio de Janeiro, v. 12, n. 2, [s.p.], 2008. Disponível em: <www. unati.uerj.br/tse/scielo.php?script $=$ sci arttext\&pid=S1809-98232008000200003\&ln $\mathrm{g}=$ pt\&nrm=iso $>$. Acesso em: 17 out. 2008.

MATSUDO, S. M. Osteoporose e a atividade física. Revista Brasileira de Ciência e Movimento, Brasília, v. 5, n. 3, p. 33-60, 1991.

MAZO, G. Z. Atividade física e qualidade de vida de mulheres idosas. 2003. Tese (Doutorado em Educação Física) - Faculdade de Ciências do Desporto e de Educação Física, Universidade do Porto. Porto, 2003.

NAHAS, M. V. Atividade física como fator de qualidade de vida. Artus - Revista de Educação Física e Desportos, Rio de Janeiro, v. 13, n. 1, p. 21-27, 1997.

PACHECO, M. D. A. et al. Qualidade de vida e performance em idosos: estudo comparativo. Saúde em Revista, Piracicaba, v. 7, n. 17, p. 47-52, 2005.

PASCOAL, M.; SANTOS, D. S. A.; VAN DEN BROEK, V. Qualidade de vida, terceira idade e atividades físicas. Motriz, Rio Claro, v. 12, n. 3 p. 217-228, 2006.

PIERON, M. Estilo de vida, prática de atividades físicas e esportivas, qualidade de vida. Fitness \& Performance Journal, Rio de Janeiro, v. 3, n. 1, p. 10-18, 2004.

PINHO, S. T. et al. A hidroginástica na terceira idade. Lecturas: Educacion Física y Deportes, a. 11, n. 102, [s.p.], 2006; Disponível em: <www.efdeportes.com/efd102/hidrog. htm>. Acesso em: 12 jan. 2007.

RAMOS, M. P. Apoio social e saúde entre idosos. Sociologias, Porto Alegre, [s.v.], n. 7, p. 156-175, 2002.

RASIA, J. et al. A relação do sobrepeso e obesidade com desconfortos musculoesqueléticos de mulheres pós-menopausa. Revista 
Brasileira de Ciência do Envelhecimento Humano, Passo Fundo, v. 4, n. 1, p. 28-38, jan./jun. 2007.

ROSA, M. F. et al. Efeito do período de interrupção de atividades aquáticas na aptidão funcional de idosas. Revista Brasileira de Cineantropometria e Desempenho Humano, Florianópolis, v. 10, n. 3, p. 237-242, 2008.

SILVA, K. M. S. et al. A influência da obesidade na capacidade funcional de mulheres acima de 51 anos. Revista Brasileira de Obesidade, Nutrição e Emagrecimento, São Paulo, v. 1, n. 1, p. 31-38, 2007.

SOARES, T. M. Estilo de vida e postura corporal em idosas. 2002. Dissertação (Mestrado em Educação Física) - Centro de Desportos, Universidade Federal de Santa Catarina. Florianópolis, 2002.

TEIXEIRA, C. Hidroginástica na reabilitação vestibular de idosos com queixas de tontura. 2008. Dissertação (Mestrado em Distúrbios da Comunicação Humana) - Centro de Ciências da Saúde, Universidade Federal de Santa Maria. Santa Maria, 2008.

TEIXEIRA, C. S.; PEREIRA, E. F.; ACOSTA, M. A. Quem são os idosos participantes do $4^{\circ}$ ACAMPAVIDA. Caderno Adulto do Núcleo Integrado de Estudo e Apoio a Terceira Idade, Centro de Educação Física e Desporto da Universidade Federal de Santa Maria, Santa Maria, n. 7, p. 19-32, 2003.

TEIXEIRA, C. S. et al. Equilíbrio corporal e exercícios físicos: uma investigação com mulheres idosas praticantes de diferentes modalidades. Acta Fisiátrica, São Paulo, v. 15, n. 3, p. 154-157, 2008.

VARGAS, L. A. M.; VARGAS NETO, F. X. Atividade física, terceira idade, saúde e longevidade. Ciência em Movimento, Porto Alegre, v. 4, n. 4, p. 30-35, 2000.

WAGORN, Y.; THÉBERGE, S.; ORBAN, W. R. Manual de ginástica e bem-estar para a terceira idade. São Paulo: Marca Zero, 1993. 